\title{
Sulfonamide Synthesis through Electrochemical Oxidative Coupling of Amines and Thiols
}

\author{
Gabriele Laudadio, ${ }^{\dagger}$ Efstathios Barmpoutsis, ${ }^{\dagger}$ Christiane Schotten, $^{\dagger, \S}$ Lisa Struik, $^{\dagger}$ Sebastian Govaerts, $^{\dagger}$ \\ Duncan L. Browne, ${ }^{\S}{ }^{\circ}$ and Timothy Noël*, ${ }^{\dagger}$ \\ ${ }^{\dagger}$ Micro Flow Chemistry and Synthetic Methodology, Department of Chemical Engineering and Chemistry, Eindhoven University of \\ Technology, Het Kranenveld, Bldg 14 - Helix, 5600 MB Eindhoven, The Netherlands \\ ${ }^{\S}$ School of Chemistry, Cardiff University, Main Building, Park Place, Cardiff CF10 3 AT, United Kingdom
}

\section{Supporting Information}

ABSTRACT: Sulfonamides are key motifs in pharmaceuticals and agrochemicals, spurring the continuous development of novel and efficient synthetic methods to access these functional groups. Herein, we report an environmentally benign electrochemical method which enables the oxidative coupling between thiols and amines, two readily available and inexpensive commodity chemicals. The transformation is completely driven by electricity, does not require any sacrificial reagent or additional catalysts and can be carried out in only $5 \mathrm{~min}$. Hydrogen is formed as a benign byproduct at the counter electrode. Owing to the mild reaction conditions, the reaction displays a broad substrate scope and functional group compatibility.

W hile rare in natural products, ${ }^{1}$ sulfonamides are valuable structural motifs in medicinal and agrochemical agents due to their chemical and metabolic stability, enhanced crystallinity, carboxyl bioisosterism, and high level of biological activity. The classical approach to prepare this moiety involves the reaction between amine nucleophiles and sulfonyl chlorides (Figure 1A). ${ }^{2,3}$ However, sulfonyl chlorides are not widely available and are toxic, unstable reagents. Their preparation is cumbersome and requires a combination of oxidizing and chlorinating reagents. ${ }^{4}$ Also, one pot procedures have been developed to prepare sulfonamides. ${ }^{5,6}$ More recently, a synthetically useful copper-catalyzed approach was reported which combines aryl boronic acids, amines and 1,4diazabicyclo[2.2.2] octane bis(sulfur dioxide) (DABSO) as $\mathrm{SO}_{2}$ precursor. ${ }^{7}$ Despite these and other interesting approaches, ${ }^{8,9}$ the direct use of commodity chemicals such as thiols and amines to prepare sulfonamides remains a hitherto elusive goal (Figure 1B). The development of such a transformation would be particularly useful given the broad availability and the low cost of these starting materials. However, a suitable transformation would require two key steps, including an S-N bond formation and a subsequent oxidation of the sulfur atom. Here, we report an electrochemical method which is able to address this specific need (Figure 1C). The use of electrochemical activation not only permits the union of these stubborn coupling partners, but also enables this transformation to be carried out under extremely mild conditions (room temperature, no hazardous reagents required) and to

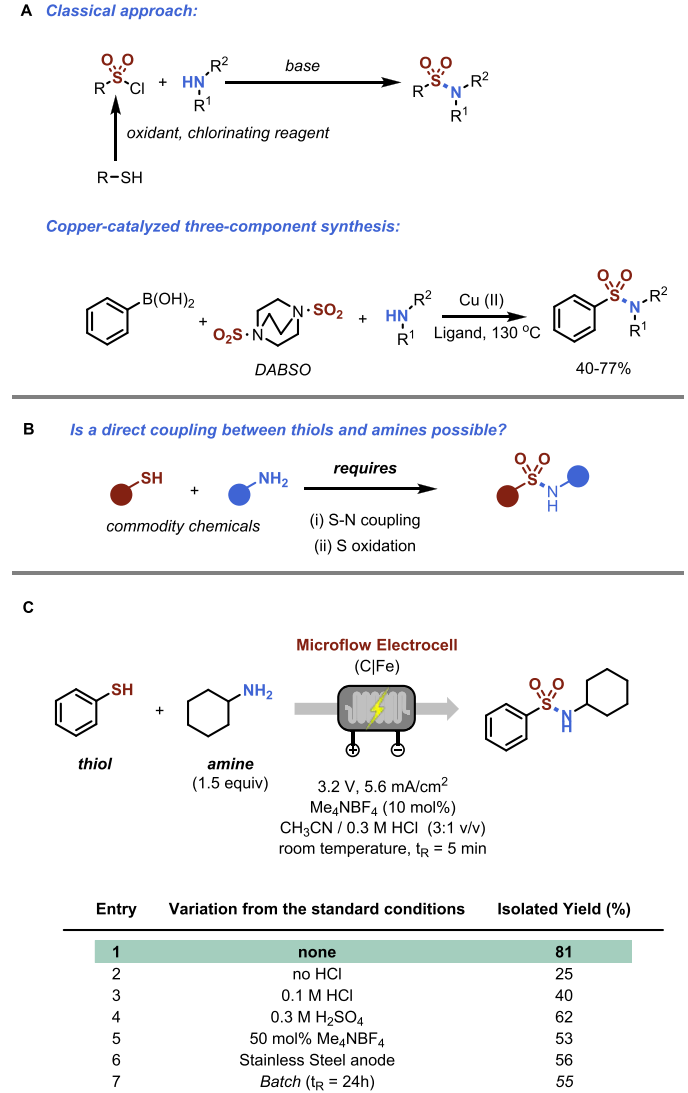

Figure 1. Development of a direct synthesis of sulfonamides starting from thiols and amines. (A) Established routes toward sulfonamides requiring prefunctionalized reagents. (B) Direct use of commodity chemicals to yield sulfonamides requires two steps. (C) Optimization of the electrochemical sulfonamide synthesis using thiophenol and cyclohexylamine. Reaction Conditions (Entry 1): thiophenol (2 mmol), cyclohexylamine (3.0 mmol), $\mathrm{Me}_{4} \mathrm{NBF}_{4}(0.2 \mathrm{mmol})$, $\mathrm{CH}_{3} \mathrm{CN} / 0.3 \mathrm{M} \mathrm{HCl}(20 \mathrm{~mL}, 3: 1 \mathrm{v} / \mathrm{v}), \mathrm{C}$ anode/Fe cathode, $5 \mathrm{~min}$ residence time, $700 \mu \mathrm{L}$ reactor volume.

avoid the use of transition metal catalysis. ${ }^{10-13}$ Hence, the electrochemical approach toward sulfonamides described herein follows the important driver to develop more

Received: February 28, 2019

Published: March 25, 2019 

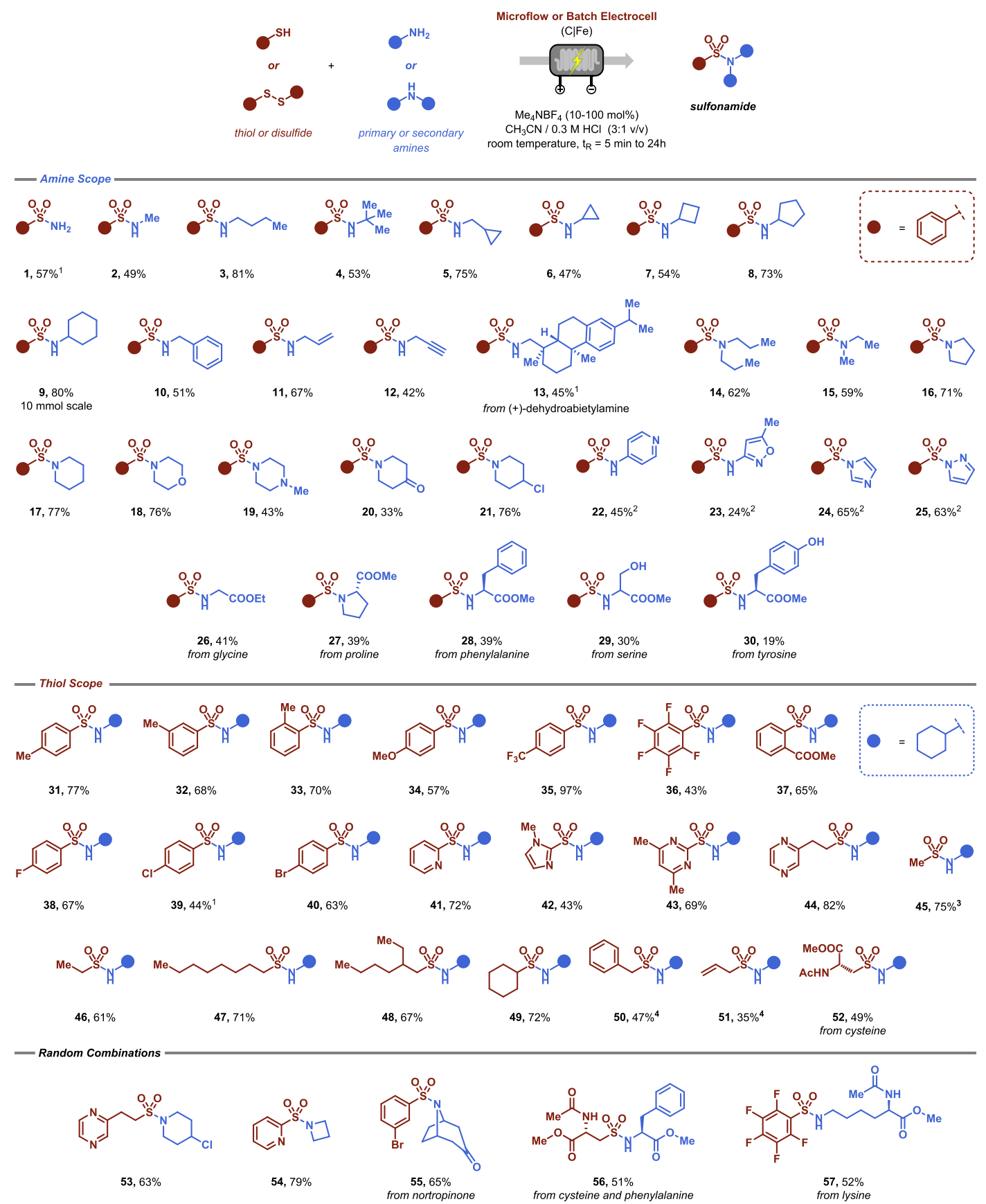

Figure 2. Synthesis of sulfonamides. Substrate scope for the electrochemical sulfonamide synthesis by direct anodic coupling of thiols and amines. All yields are isolated and reproduced at least two times. Reaction conditions: thiol (2 mmol), amine ( $3.0 \mathrm{mmol}), \mathrm{Me}_{4} \mathrm{NBF}_{4}(0.2 \mathrm{mmol}), \mathrm{CH}_{3} \mathrm{CN} /$ $0.3 \mathrm{M} \mathrm{HCl}(20 \mathrm{~mL}, 3: 1 \mathrm{v} / \mathrm{v}), \mathrm{C}$ anode/Fe cathode, $5 \mathrm{~min}$ residence time, $700 \mu \mathrm{L}$ reactor volume. ${ }^{1}$ Reaction carried out in a batch reactor. Reaction conditions: thiol $(3 \mathrm{mmol})$, amine $(4.5 \mathrm{mmol}), \mathrm{Me}_{4} \mathrm{NBF}_{4}(3 \mathrm{mmol}), \mathrm{CH}_{3} \mathrm{CN} / 0.3 \mathrm{M} \mathrm{HCl}(30 \mathrm{~mL}, 3: 1 \mathrm{v} / \mathrm{v}), \mathrm{C}$ anode/Fe cathode, $24 \mathrm{~h}$ reaction time. ${ }^{2}$ Pyridine $(2.0 \mathrm{mmol})$ was added as an additive. ${ }^{3}$ The corresponding disulfide was used as starting material. ${ }^{4} 10$ min of residence time instead of $5 \mathrm{~min}$.

sustainable synthetic methods, while addressing a fundamental and hitherto unanswered synthetic challenge of preparing sulfonamides from thiol and amine feedstock inputs. ${ }^{14}$

We began our investigations by establishing suitable reaction conditions for the coupling between thiophenol and cyclohexyl amine (Figure 1C). We used an electrochemical microflow reactor to rapidly screen the different reaction variables. ${ }^{15}$ Because of the small interelectrode gap $(250 \mu \mathrm{m})$, the high mass transfer and the large electrode surface to volume ratio, intensified reaction conditions are observed in this reactor. ${ }^{16,17}$
Indeed, after extensive screening of conditions, the reaction could be completed in only 5 min furnishing the targeted sulfonamide in good isolated yield as shown in entry 1 . The reaction requires only a small excess of amine (1.5 equiv), 10 mol \% of $\mathrm{Me}_{4} \mathrm{NBF}_{4}$ as electrolyte and can be carried out in a $3: 1(\mathrm{v} / \mathrm{v})$ mixture of $\mathrm{CH}_{3} \mathrm{CN} / 0.3 \mathrm{M} \mathrm{HCl}$ at room temperature using a combination of inexpensive graphite/stainless steel electrodes. In the absence of acid or at lower concentrations, lower isolated yields are obtained (entries 2 and 3 ). Switching to sulfuric acid gave slightly lower yields compared to 
A

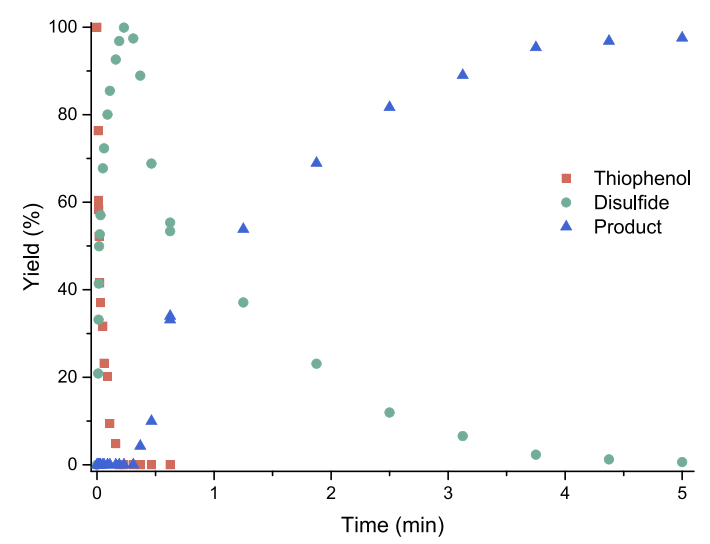

B

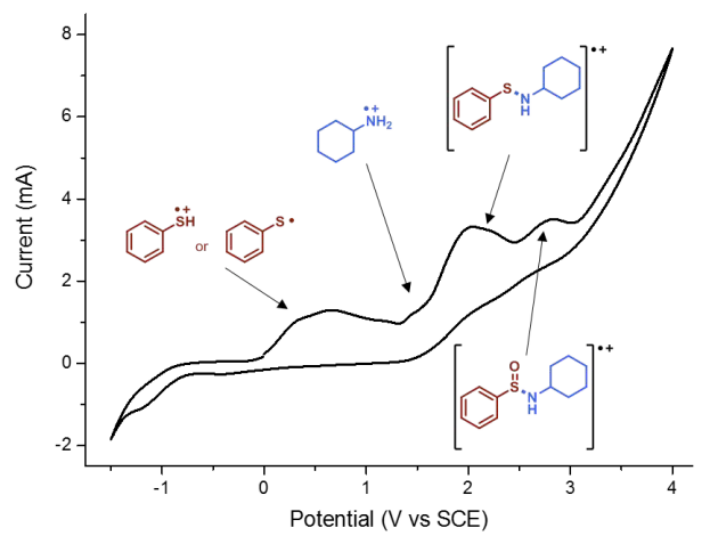

C

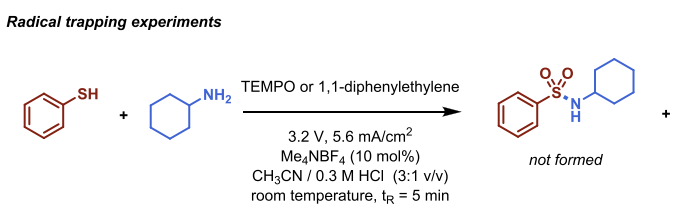

Intermediates subjected to reaction conditions<smiles>NC1CCCCC1</smiles>

disulfide<smiles>c1ccc(SNC2CCCCC2)cc1</smiles>

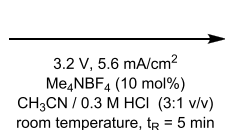
$3.2 \mathrm{~V}, 5.6 \mathrm{~mA} / \mathrm{cm}^{2}$ $\mathrm{Me}_{4} \mathrm{NBF}_{4}(10 \mathrm{~mol} \%)$ $\mathrm{CH}_{3} \mathrm{CN} / 0.3 \mathrm{M} \mathrm{HCl}(3: 1 \mathrm{v} / \mathrm{v})$

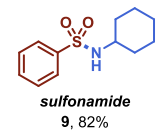
room temperature, $t_{\mathrm{p}}=5 \mathrm{~min}$
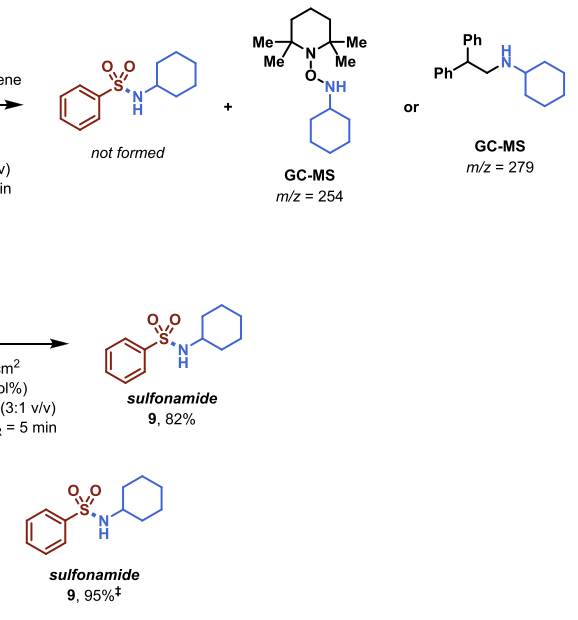

D

Figure 3. Mechanistic investigation of the electrochemical sulfonamide synthesis. (A) Kinetic experiment. (B) Cyclic voltammetry. See Supporting Information for more details. (C) Radical trapping experiments and intermediate evaluation. ${ }^{\ddagger}$ GC Yield (biphenyl as internal standard). (D) Proposed mechanism.

hydrochloric acid (entry 4). Interestingly, a higher electrolyte concentration led to lower yields, presumably due the formation of an electrolyte film on the graphite electrode (entry 5). ${ }^{18}$ Other anode materials were less efficient (entry 6). Notably, carrying out the new transformation in a batch electrochemical cell was also possible but required longer reaction times $(24 \mathrm{~h})$ and an increased electrolyte loading. The increase in electrolyte loading is required to compensate for the higher ohmic drop with increasing interelectrode distances, while the longer reaction times can be attributed to a lower electrode-to-volume ratio and mass transfer limitations. ${ }^{19}$

With optimal conditions established, we examined the generality of our electrochemical transformation. In most cases we used flow processing to obtain optimal yields. However, in certain circumstances, e.g. when longer reaction times are required or when insoluble starting materials are used, conventional batch techniques proved effective to obtain the targeted sulfonamide. As shown in Figure 2, a wide variety of structurally and electronically distinct amines and thiols can be engaged in this transformation. Free amine sulfonamides can be prepared with ammonia (1); these compounds have great value in drug discovery programs but can also be readily modified through arylation using a $\mathrm{C}-\mathrm{N}$ cross coupling strategy or more traditional coupling alkylation reactions. ${ }^{20}$
Furthermore, a variety of primary amines are competent coupling partners in this protocol, including methylamine (2), butylamine (3), tert-butylamine (4), cyclopropanemethylamine (5), cyclopropylamine (6), cyclobutylamine (7), cyclopentylamine (8), cyclohexylamine (9) and benzylamine (10), delivering the targeted products in good isolated yields. The reaction conditions are readily scaled in flow as demonstrated for sulfonamide 9, which was carried out on a $10 \mathrm{mmol}$ scale. Allylamine (11) and propargylamine (12) are also amenable to the reaction conditions and gave synthetically useful yields. These sulfonamides are particularly interesting for further synthetic diversification and use in bioconjugation processes using strategies such as click chemistry. ${ }^{21}$ The coupling of more structurally complex primary amines, such as (+)-dehydroabietylamine (13), is also readily accomplished using this electrochemical method. In addition, a diverse set of secondary amines, such as dipropylamine (14), methylethylamine (15), pyrrolidine (16), piperidine (17), morpholine (18), Nmethylpiperazine (19), 4-piperidone (20) and 4-chloropiperidine (21), were effective substrates for this protocol. In contrast, heteroarylamines proved to be challenging substrates resulting only in trace amounts of product. However, by adding an equivalent of pyridine as an electron-mediator, ${ }^{22}$ these substrates became competent coupling partners allowing 
the conversion of 4-aminopyridine (22), 3-amino-4-methylisoxazole (23), as well as imidazole (24) and pyrazole (25) to the corresponding sulfonamide. As a further demonstration of the utility of this method, we considered functionalizing amino acids, which would allow the preparation of nonproteinogenic building blocks for the discovery of new therapeutic peptides. The preparation of a diverse set of sulfonamides derived from glycine (26), proline (27), phenylalanine (28), serine (29) and tyrosine (30) was successful and further demonstrates the functional group tolerance of this electrochemical method. Notably, no racemization of the chiral centra was observed under these reaction conditions (see Supporting Information).

Similarly, we investigated the breadth of thiols that are compatible with the reaction conditions by coupling them with cyclohexylamine. Thiophenols bearing electron-neutral (3133), -donating (34) and -withdrawing substituents (35-37) were all tolerated. The reaction is not particularly sensitive to sterical hindrance as ortho-substituted thiophenols (33, 36 and 37) displayed similar yields to those with meta- (32) or parasubstituents (e.g., 31, 34-35). Interestingly, halogenated thiophenols $(36,38-40)$ were viable substrates as well, providing functional handles for further modification using classical cross-coupling methods. Heterocyclic thiols, such as 2mercaptopyridine (41), methimidazole (42), 2-mercapto-4,6dimethylpyrimidine (43) and pyrazineethanethiol (44), were adequate coupling partners furnishing the targeted compounds in good to excellent yields. Notably, while methanethiol is a colorless, flammable and toxic gas with a repulsive smell, we could use the corresponding disulfide as alternative input feed to furnish the desired sulfonamide (45) in $75 \%$ isolated yield. Other aliphatic thiols were equally effective, including ethanethiol (46), octanethiol (47), 2-ethyl-hexanethiol (48), cyclohexylthiol (49), benzylthiol (50), and allylthiol (51). The use of cysteine furnished the targeted compound in $49 \%$ yield (52). While previous examples kept one of the reaction partners constant, random variations are possible as shown by examples 53-57. Biologically interesting amines, such as azetidine (54) and nortropinone (55), displayed excellent reactivity. Interestingly, we were able to couple cysteine with phenylalanine via the electrochemical sulfonylative coupling in good isolated yield $(56,51 \%)$ providing opportunities for peptide modification. We also found that lysine (57) functioned well as a coupling partner in our electrochemical sulfonamide protocol.

A number of additional experiments were carried out to elucidate the reaction mechanism of the electrochemical sulfonamide synthesis (Figure 3). Kinetic experiments revealed that within the first $20 \mathrm{~s}$ of the reaction the thiol substrate is completely converted via anodic oxidation to the corresponding disulfide (Figure 3A). ${ }^{23}$ Indeed, we found that disulfides were equally competent coupling partners compared to the parent thiol substrates, providing opportunities to circumvent the use of some of the most odorous thiols (Figure 3C). The disulfide is consumed within $5 \mathrm{~min}$ and the corresponding sulfonamide is formed, albeit at a slightly different rate. This hints to the fact that the sulfonamide formation occurs via several intermediate steps.

Following the electrochemical disulfide formation, the amine is oxidized to the radical cation. ${ }^{24,25}$ Adding TEMPO or 1,1diphenylethylene as radical scavengers completely shuts down the sulfonamide formation and the corresponding radical adducts were found via GC-MS, substantiating the generation of aminium radical intermediates which are key in this electrochemical process (Figure 3C). The aminium radical intermediate subsequently reacts with the disulfide to generate the sulfenamide. Interestingly, no Shono-type oxidation of the amines was observed under these reaction conditions. ${ }^{26}$ Next, two consecutive oxidation steps of the sulfenamide take place and the targeted sulfonamide is formed via a sulfinamide intermediate. ${ }^{27}$ Indeed, we were able to isolate the sulfenamide intermediate and, by subjecting this compound to our reaction protocol, the corresponding sulfonamide was generated effectively (Figure 3C).

To gain further insight into the electrochemical reaction process, including information on the oxidation potentials of the different coupling partners and intermediates, a series of cyclovoltammetry studies were carried out (Figure 3B). As suggested by our experimental results, the disulfide is formed first with an oxidation potential $\left(E_{\mathrm{ox}}\right)$ of $\sim 0.5 \mathrm{~V}$. Next, the amine radical is generated at $\sim 1.5 \mathrm{~V}$. After generation of the sulfenamide, two consecutive oxidations are observed at $\sim 2.0$ and $\sim 2.6 \mathrm{~V}$ forming the corresponding sulfonamide. At the counterelectrode, hydrogen evolution as a benign byproduct was clearly observed. ${ }^{28}$

We anticipate that this green and mild synthetic protocol to prepare sulfonamides will find use in both academic and industrial settings. Furthermore, we postulate that the convenience with which this electrochemical method activates commodity chemicals will inspire further advances in the use of electrochemistry to enable challenging yet hitherto unanswered synthetic transformations.

\section{ASSOCIATED CONTENT}

\section{S Supporting Information}

The Supporting Information is available free of charge on the ACS Publications website at DOI: 10.1021/jacs.9b02266.

Data and materials availability: additional optimization, mechanistic data, experimental procedures and analytical data $\left({ }^{1} \mathrm{H},{ }^{19} \mathrm{~F}\right.$ and ${ }^{13} \mathrm{C}$ NMR, MS $)$ for all new compounds (PDF)

\section{AUTHOR INFORMATION}

\section{Corresponding Author}

*T.Noel@tue.nl

ORCID $\odot$

Gabriele Laudadio: 0000-0002-2749-8393

Duncan L. Browne: 0000-0002-8604-229X

Timothy Noël: 0000-0002-3107-6927

\section{Notes}

The authors declare no competing financial interest.

\section{ACKNOWLEDGMENTS}

We acknowledge financial support from the Dutch Science Foundation (NWO) for a VIDI grant for T.N. (SensPhotoFlow, No. 14150). C.S. and D.L.B. thank the Royal Society of Chemistry for a Researcher Mobility Grant. S.G. is grateful to the European Union for receiving Erasmus+ grant.

\section{REFERENCES}

(1) Petkowski, J. J.; Bains, W.; Seager, S. Natural Products Containing a Nitrogen-Sulfur Bond. J. Nat. Prod. 2018, 81, 423-446.

(2) Forster, M. O.; Kunz, E. CLXIII.-Studies in the camphane series. Part XXXV. Isomeric hydrazoximes of camphorquinone, and some derivatives of aminocamphor. J. Chem. Soc., Trans. 1914, 105, $1718-1733$. 
(3) Autenrieth, W.; Koburger, J. Ueber die Einwirkung aromatischer Amine auf Aethylendisulfochlorid und über Vinylsulfonderivate. Ber. Dtsch. Chem. Ges. 1903, 36, 3626-3634.

(4) Schmitt, A.-M. D.; Schmitt, D. C. Chapter 13. Synthesis of Sulfonamides. In Synthetic Methods in Drug Discovery: Volume 2; Blakemore, D. C., Doyle, P. M., Fobian, Y. M., Eds.; RSC Drug Discovery Series; Royal Society of Chemistry, 2016; pp 123-138.

(5) Sohrabnezhad, S.; Bahrami, K.; Hakimpoor, F. High yielding protocol for direct conversion of thiols to sulfonyl chlorides and sulfonamides. J. Sulfur Chem. 2019, 1.

(6) Bahrami, K.; Khodaei, M. M.; Soheilizad, M. Direct Conversion of Thiols to Sulfonyl Chlorides and Sulfonamides. J. Org. Chem. 2009, 74, 9287-9291.

(7) Chen, Y.; Murray, P. R. D.; Davies, A. T.; Willis, M. C. Direct Copper-Catalyzed Three-Component Synthesis of Sulfonamides. J. Am. Chem. Soc. 2018, 140, 8781-8787.

(8) Hofman, K.; Liu, N.-W.; Manolikakes, G. Radicals and Sulfur Dioxide: A Versatile Combination for the Construction of SulfonylContaining Molecules. Chem. - Eur. J. 2018, 24, 11852-11863.

(9) Mulina, O. M.; Ilovaisky, A. I.; Terent'ev, A. O. Oxidative Coupling with S-N Bond Formation. Eur. J. Org. Chem. 2018, 2018, 4648-4672.

(10) Wiebe, A.; Gieshoff, T.; Möhle, S.; Rodrigo, E.; Zirbes, M.; Waldvogel, S. R. Electrifying Organic Synthesis. Angew. Chem., Int. Ed. 2018, 57, 5594-5619.

(11) Yan, M.; Kawamata, Y.; Baran, P. S. Synthetic Organic Electrochemical Methods Since 2000: On the Verge of a Renaissance. Chem. Rev. 2017, 117, 13230-13319.

(12) Yoshida, J.-i.; Kataoka, K.; Horcajada, R.; Nagaki, A. Modern Strategies in Electroorganic Synthesis. Chem. Rev. 2008, 108, 22652299.

(13) Moeller, K. D. Synthetic Applications of Anodic Electrochemistry. Tetrahedron 2000, 56, 9527-9554.

(14) Anastas, P.; Eghbali, N. Green Chemistry: Principles and Practice. Chem. Soc. Rev. 2010, 39, 301-312.

(15) Laudadio, G.; de Smet, W.; Struik, L.; Cao, Y.; Noël, T. Design and application of a modular and scalable electrochemical flow microreactor. J. Flow Chem. 2018, 8, 157-165.

(16) Atobe, M.; Tateno, H.; Matsumura, Y. Applications of Flow Microreactors in Electrosynthetic Processes. Chem. Rev. 2018, 118, 4541-4572.

(17) Pletcher, D.; Green, R. A.; Brown, R. C. D. Flow Electrolysis Cells for the Synthetic Organic Chemistry Laboratory. Chem. Rev. 2018, 118, 4573-4591.

(18) Somerville, L.; Bareño, J.; Jennings, P.; McGordon, A.; Lyness, C.; Bloom, I. The Effect of Pre-Analysis Washing on the Surface Film of Graphite Electrodes. Electrochim. Acta 2016, 206, 70-76.

(19) Folgueiras-Amador, A. A.; Wirth, T. Perspectives in flow electrochemistry. J. Flow Chem. 2017, 7, 94-95.

(20) Kim, T.; McCarver, S. J.; Lee, C.; MacMillan, D. W. C. Sulfonamidation of Aryl and Heteroaryl Halides through Photosensitized Nickel Catalysis. Angew. Chem., Int. Ed. 2018, 57, 34883492.

(21) Thirumurugan, P.; Matosiuk, D.; Jozwiak, K. Click Chemistry for Drug Development and Diverse Chemical-Biology Applications. Chem. Rev. 2013, 113, 4905-4979.

(22) Francke, R.; Little, R. D. Redox catalysis in organic electrosynthesis: basic principles and recent developments. Chem. Soc. Rev. 2014, 43, 2492.

(23) Laudadio, G.; Straathof, N. J. W.; Lanting, M. D.; Knoops, B.; Hessel, V.; Noël, T. An environmentally benign and selective electrochemical oxidation of sulfides and thiols in a continuous-flow microreactor. Green Chem. 2017, 19, 4061-4066.

(24) Gieshoff, T.; Kehl, A.; Schollmeyer, D.; Moeller, K. D.; Waldvogel, S. R. Insights into the Mechanism of Anodic N-N Bond Formation by Dehydrogenative Coupling. J. Am. Chem. Soc. 2017, 139, 12317-12324.

(25) Chow, Y. L.; Danen, W. C.; Nelsen, S. F.; Rosenblatt, D. H. Nonaromatic aminium radicals. Chem. Rev. 1978, 78, 243-274.
(26) Jones, A. M.; Banks, C. E. The Shono-type electroorganic oxidation of unfunctionalised amides. Carbon-carbon bond formation via electrogenerated $\mathrm{N}$-acyliminium ions. Beilstein J. Org. Chem. 2014, 10 (1), 3056-3072.

(27) D’Oca, M. G. M.; Russowsky, D.; Canto, K.; Gressler, T.; Gonçalves, R. S. Electrochemical Oxidation of N - p -Toluenesulfinamides. Org. Lett. 2002, 4, 1763-1766.

(28) Tang, S.; Liu, Y.; Lei, A. Electrochemical Oxidative Crosscoupling with Hydrogen Evolution: A Green and Sustainable Way for Bond Formation. Chem. 2018, 4, 27-45. 